\title{
Characterization of the Inverse Exponential-Type Distribution Based on Recurrence Relations for Dual Generalized Order Statistics
}

\author{
M. A. W. Mahmoud \\ Department of Mathematics \\ Faculty of Science, \\ Al-Azhar University, Nasr City, \\ Cairo (11884), Egypt.
}

\author{
Y. Abdel-Aty \\ Department of Mathematics \\ Faculty of Science, \\ Al-Azhar University, Nasr City, \\ Cairo (11884), Egypt.
}

\author{
N. M. Mohamed \\ Department of Mathematics \\ Faculty of Science, \\ Suez University, Suez, \\ Egypt.
}

\begin{abstract}
In this paper, new recurrence relations satisfied by the single and product moments using moment generating function of dual generalized order statistics from inverse exponential-type distribution are established. Recurrence relations for single and product moments of reversed order statistics and lower record value are obtained as special cases. Further, using a recurrence relation for single moments we obtain characterization of inverse exponential-type distribution.
\end{abstract}

\section{Keywords}

Dual generalized order statistics, moment generating function, recurrence relations, single moments, product moments, inverse exponential form and characterization.

\section{INTRODUCTION}

The importance of generalized order statistics (gos) due to inclusion special cases which have been used in statistical research such as order statistics and record value, see [1]. Let $F(-)$ be absolutely continuous distribution function with probability density function $f(-)$. Further, let $n \in N, k \geq 0$ $, n \geq 2, m=\left(m_{1}, m_{2}, \cdots m_{n-1}\right) \in R^{n-1}, M_{r}=\sum_{j=r}^{n-1} m_{j}$, such that $\gamma_{r}=k+n-r+M_{r}>0$, for all $r \in\{1,2, \cdots, n-1\}$. Then $X^{\prime}(n, n, m, k), r=1,2, \cdots, n$ are called gos if their joint pdf given by:

$k\left(\prod_{j=1}^{n-1} \gamma_{j}\right)\left(\prod_{i=1}^{n-1}\left[1-F\left(x_{i}\right)\right]^{m} f\left(x_{i}\right)\right)\left[1-F\left(x_{n}\right)\right]^{k-1} f\left(x_{n}\right)$,

for

$F^{-1}\left(0^{+}\right)<x_{1} \leq x_{2} \leq \cdots \leq x_{n}<F^{-1}(1)$ of $R^{n-1}$.

[2] represents the concept of dual generalized order statistics (dgos) as follows:

Let $n \in N, k \geq 1$, be the parameters such that

$$
\gamma_{r}=k+(n-r)(m+1)>0 \text {, for all } 1,, r, n \text {. }
$$

Let $X^{\prime}(1, n, m, k), X^{\prime}(2, n, m, k) \cdots, X^{\prime}(n, n, m, k)$ be the dgos from an absolutely continuous distribution function $F(-)$ with density function $f(-)$, so, the joint probability density function (pdf) of the form

$k\left(\prod_{j=1}^{n-1} \gamma_{j}\right)\left(\prod_{i=1}^{n-1}\left[F\left(x_{i}\right)\right]^{m} f\left(x_{i}\right)\right)\left[F\left(x_{n}\right)\right]^{k-1} f\left(x_{n}\right),(1)$

for

$$
F^{-1}(1)>x_{1} \geq x_{2} \geq \cdots \geq x_{n}>F^{-1}(0)
$$

The marginal pdf of r-th dgos, $X^{\prime}(r, n, m, k)$ is

$$
f_{X^{\prime}(r, n, m, k)}(x)=\frac{C_{r-1}}{\Gamma(r)}[F(x)]^{\gamma_{r}-1} f(x) g_{m}^{r-1}(F(x)) .
$$

The joint pdf of $X(r, n, m, k)$ and $X(s, n, m, k)$, $1 \leq r \leq s \leq n, x>y$ is expressed from (1) as

$$
\begin{aligned}
& f_{X^{\prime}(r, n, m, k), X^{\prime}(s, n, m, k)}(x, y) \\
& =\frac{C_{s-1}}{\Gamma(r) \Gamma(s-r)}[F(x)]^{m} f(x) g_{m}^{r-1}(F(x)) \\
& \quad \times\left[h_{m}(F(y))-h_{m}(F(x))\right]^{s-r-1}[F(y)]^{\gamma_{s}-1} f(y),
\end{aligned}
$$

where

$$
\Gamma(r)=\int_{0}^{\infty} u^{r-1} \exp (-u) d u
$$

$$
C_{r-1}=\prod_{i=1}^{r} \gamma_{i}
$$




$$
h_{m}(x)= \begin{cases}\frac{-1}{m+1} x^{m+1}, & m \neq-1 \\ -\ln x, & m=-1 .\end{cases}
$$

and

$$
g_{m}(x)=h_{m}(x)-h_{m}(1), x \in[0,1)
$$

Since $X^{\prime}(0, n, m, k) \rightarrow 0$ then $X^{\prime}(n+1, n, m, k)=0$. If $m=0, k=1$, then $X(r, n, m, k)$ reduces to the $(n-r+1)-t h$ reversed order statistics, $\left(X_{n-r+1: n}\right)$ from the sample $X_{1}, X_{2}, \cdots, X_{n}$ and when $m=-1$, then $X(r, n, m, k)$ reduces to the k-lower record value [3].

[1] dealt with generalized order statistics from kumaraswamy distributibution and its characterization. Recurrence relations for moment generating functions of order statistics are established by [4]. [2] established moments of lower generalized order statistics from exponentiated Pareto distribution and its characterization. Recurrence relations for single and product moments of dual generalized order statistics from the inverse Weibull distribution are derived by [5]. [6] have established recurrence relations for moments of dual generlized order statistics from exponentiated Weibull distribution. [7] obtained recurrence relations for momet and conditional moment generating functions of gos based on random samples drawn from a population whose distribution is a member of a doubly truncated class of distributions.

Consider the cummulative distribution function (cdf) is:

$$
F(x)=\exp [-\lambda(x)], x \geq 0,
$$

where $\lambda(x)$ is a non-negative, continuous, monotone decreasing, differentiable function of $x$ such that $\lambda(x) \rightarrow 0$ as $x \rightarrow \infty$ and $\lambda(x) \rightarrow \infty$ as $x \rightarrow 0^{+}$. This family contains many distributions such as inverse Weibull distribution, inverse exponential distribution and inverse Rayleigh distribution.

The probability density function (pdf) is given by:

$$
f(x)=-\lambda(x) \exp [-\lambda(x)], x \geq 0 .
$$

Therefore, from (4) and (5), we have

$$
F(x)=-\frac{1}{\lambda^{\prime}(x)} f(x) .
$$

\begin{tabular}{|c|c|c|}
\hline Distribution & cdf & $\lambda(x)$ \\
\hline $\begin{array}{l}\text { inverse } \\
\text { Weibull }\end{array}$ & $\exp \left(-(\alpha x)^{-\beta}\right)$ & $\frac{\alpha^{-\beta}}{x^{\beta}}, x>0, \alpha, \beta>0$. \\
\hline $\begin{array}{c}\text { inverse } \\
\text { exponential }\end{array}$ & $\exp \left(-(\alpha x)^{-1}\right)$ & $\frac{1}{\alpha x}, x>0, \beta=1$ \\
\hline $\begin{array}{l}\text { inverse } \\
\text { Rayleigh }\end{array}$ & $\exp \left(-(\alpha x)^{-2}\right)$ & $\frac{1}{(\alpha x)^{2}}, x>0, \alpha, \beta=2$ \\
\hline
\end{tabular}

The following table gives some distributions with proper choice of $\lambda(x)$ as examples:-

\section{CHARACTERIZATION BASED ON RECURRENCE RELATIONS FOR SINGLE MOMENT GENERATING FUNCTIONS OF DGOS}

The single moment generating function of dgos can be obtained, for $a \geq 1$, from Using (2), we have when $m \neq-1$

$$
\begin{aligned}
& M_{r ; n: m: k}^{(a)}(t)=E\left[e^{t X_{(r, n, m, k)}^{(a)}}\right] \\
& =\frac{C_{r-1}}{\Gamma(r)} \int_{0}^{\infty} e^{t x^{a}}(F(x))^{\gamma_{r}-1} f(x) g_{m}^{r-1}(F(x)) d x,
\end{aligned}
$$

for simplicity $X_{(r, m, n, k)}=x$.

Relation 1 Let $X$ be a random variable, then for integers $a$ such that $a \geq 1$, the following recurrence relation is satisfied iff $X$ has cdf (4).

$$
M_{r ; n: m: k}^{(a)}(t)-M_{r-1 ; n: m: k}^{(a)}(t)=\frac{a t}{\gamma_{r}} E\left[\frac{X_{(r, n: m: k)}^{(a-1)} e^{t X\left(\begin{array}{l}
(a) \\
(r, n: m: k)
\end{array}\right.}}{\lambda\left(X_{(r, n: m: k)}^{\prime}\right)}\right]
$$

Proof: Using(7) and (2), we have

$$
M_{r ; n: m: k}^{(a)}(t)=\frac{C_{r-1}}{\Gamma(r)} \int_{0}^{\infty} e^{t x^{a}}(F(x))^{\gamma_{r}-1} f(x) g_{m}^{r-1}(F(x)) d x, \text { (9) }
$$

integrating by parts, we get

$$
\begin{aligned}
& M_{r ; n: m: k}^{(a)}(t)-M_{r-1 ; n: m: k}^{(a)}(t) \\
& =-\frac{a t C_{r-1}}{\gamma_{r} \Gamma(r)} \int_{0}^{\infty} x^{a-1} e^{t x^{a}}(F(x))^{\gamma_{r}} g_{m}^{r-1}(F(x)) d x,
\end{aligned}
$$

upon using (1.6) in (2.4), we obtain

$$
M_{r ; n: m: k}^{(a)}(t)-M_{r-1 ; n: m: k}^{(a)}(t)
$$


$=\frac{a t C_{r-1}}{\gamma_{r} \Gamma(r)} \int_{0}^{\infty} \frac{x^{a-1}}{\lambda^{\prime}(x)} e^{t x^{a}}(F(x))^{\gamma_{r}-1} f(x) g_{m}^{r-1}(F(x)) d x$,

so, we have the result.

Conversely, if the characterizing condition (8) holds, then from (2) and (6), we have

$\frac{C_{r-1}}{\Gamma(r)} \int_{0}^{\infty} e^{t x^{a}}(F(x))^{\gamma_{r}-1} f(x) g_{m}^{r-1}(F(x)) d x$

$=\frac{C_{r-2}}{\Gamma(r)} \int_{0}^{\infty} e^{t x^{a}}(F(x))^{\gamma_{r}+m} f(x) g_{m}^{r-2}(F(x)) d x+\frac{a t C_{r-1}}{\gamma_{r} \Gamma(r)}$

$\times \int_{0}^{\infty} \frac{x^{a-1}}{\lambda^{\prime}(x)} e^{t x^{a}}(F(x))^{\gamma_{r}-1} f(x) g_{m}^{r-1}(F(x)) d x,(11)$

integrating the first integral on the right hand side of Equation (11) by parts, we get

$\frac{C_{r-1}}{\Gamma(r)} \int_{0}^{\infty} e^{t x^{a}}(F(x))^{\gamma_{r}-1} f(x) g_{m}^{r-1}(F(x)) d x$

$=\frac{a t C_{r-2}}{\Gamma(r)} \int_{0}^{\infty} x^{a-1} e^{t x^{a}}(F(x))^{\gamma_{r}} g_{m}^{r-1}(F(x)) d x$

$+\frac{\gamma_{r} C_{r-2}}{\Gamma(r)} \int_{0}^{\infty} e^{t x^{a}}(F(x))^{\gamma_{r}-1} f(x) g_{m}^{r-1}(F(x)) d x+\frac{a t C_{r-2}}{\gamma_{r} \Gamma(r)}$

$\times \int_{0}^{\infty} \frac{x^{a-1}}{\lambda^{\prime}(x)} e^{t x^{a}}(F(x))^{\gamma_{r}-1} f(x) g_{m}^{r-1}(F(x)) d x$,

which reduces to

$$
\begin{aligned}
\frac{a t C_{r-1}}{\gamma_{r} \Gamma(r)} \int_{0}^{\infty} x^{a-1} e^{t x^{a}}(F(x))^{\gamma_{r}} g_{m}^{r-1}(F(x)) & \\
& \times\left\{F(x)+\frac{1}{\lambda^{\prime}(x)} f(x)\right\} d x=0 .
\end{aligned}
$$

Now applying a generalization of the Muntz-Szasz Theorm to (13) [8], we get

$$
F(x)=-\frac{1}{\lambda^{\prime}(x)} f(x), x \geq 0 .
$$

\section{SPECIAL CASES}

(1) By differentiating both sides of Condition (8) with respect to $t$ and then setting $t=0$, we obtain the following recurrence relation for single moment of dgos: $\mu_{r ; n: m: k}^{(a)}-\mu_{r-1 ; n: m: k}^{(a)}=\frac{a}{\gamma_{r}} E\left[\frac{X_{r, n: m: k}^{(a-1)}}{\lambda\left(x_{r, m: n: n}\right)}\right]$,

where

$$
\mu_{r ; n: m: k}^{(a)}=E\left(X_{r, n: m: k}^{(a)}\right) .
$$

(2) Putting $m=0, k=1$ in (8), we obtain the recurrence relations of reversed order statistics as follows:

$M_{r: n}^{(a)}(t)-M_{r-1: n}^{(a)}(t)=\frac{a t}{n-r+1} E\left[\frac{X_{r: n}^{(a-1)} e^{t X_{r: n}^{(a)}}}{\lambda^{\prime}\left(X_{r: n}\right)}\right]$,

$\mu_{r: n}^{(a)}-\mu_{r-1: n}^{(a)}=\frac{a}{n-r+1} E\left[\frac{X_{r: n}^{(a-1)}}{\left.\lambda X_{r: n}^{\prime}\right)}\right]$,

where $\gamma_{r}=n-r+1, X_{r, n: m: k}=X_{r: n}$

(3) Setting $m=-1, k=1$ in (8), we get recurrence relation for single moment of lower record values in the form:

$M_{L(r)}^{(a)}(t)-M_{L(r-1)}^{(a)}(t)=a t E\left[\frac{X_{L(r)}^{(a-1)} e^{t X_{L(r)}^{(a)}}}{\lambda^{\prime}\left(X_{L(r)}\right)}\right]$,

$M_{L(r)}^{(a)}-M_{L(r-1)}^{(a)}=a E\left[\frac{X_{L(r)}^{(a-1)}}{\lambda^{\prime}\left(X_{L(r)}\right)}\right]$,

where $\gamma_{r}=1, X_{r, n: m: k}=X_{L(r)}$.

\section{CHARACTERIZATION BASED ON RECURRENCE RELATIONS FOR CONDITIONAL MOMENT GENERATING FUNCTIONS OF DGOS}

On using (3), the conditional distribution function of $X(s, n, m, k)$ given $X(s, n, m, k)$, is given by:

$$
\begin{aligned}
& f\left[X_{(s, n, m, k)} \mid X_{(r, n, m, k)}=y\right]=\frac{C_{s-1}}{C_{r-1} \Gamma(s-r)}[F(y)]^{m-\gamma_{r}+1} \\
& \times\left[h_{m}(F(x))-h_{m}(F(y))\right]^{s-r-1}[F(x)]^{\gamma_{s}-1} f(x) .(17)
\end{aligned}
$$

Relation 2 Let $X$ be a random variable, $r, s$ be two integers such that $1, r, s, n, m$ and $k$ be real numbers such that $m \geq-1, k \geq 1$. Then for integers $a$ such that $a \geq 1$, the following recurrence relation is satisfied iff $X$ has the $\operatorname{cdf}(4)$. 


$$
\begin{aligned}
& M_{\left.X_{(s, n: m: k)}^{(a)}\right|_{(r, n: m: k)}}(t \mid y)-M_{\left.X_{(s-1, n: m: k)}^{(a)}\right|_{(r, n: m: k)}}(t \mid y) \\
& =\frac{a t}{\gamma_{s}} E\left[\frac{X_{(s, n: m: k)}^{(a-1)} e^{t X_{(s, n: m: k)}^{(a)}}}{\lambda\left(X_{s, n: m: k}^{\prime}\right)} \mid X_{(r, n: m: k)}=y\right] .
\end{aligned}
$$

Proof Using (17), we get

$$
\begin{aligned}
& M_{\left.X_{(s, n: m: k)}^{(a)}\right|_{(r, n: m: k)}}(t \mid y)=E\left[e^{t X_{(s, n: m: k)}^{a}} \mid X_{r, n: m: k}=y\right] \\
& =\frac{C_{s-1}[F(y)]^{m-\gamma_{r}+1}}{C_{r-1} \Gamma(s-r)}
\end{aligned}
$$$$
\times \int_{0}^{x} e^{t x^{a}}\left[h_{m}(F(x))-h_{m}(F(y))\right]^{s-r-1} f(x)[F(x)]^{\gamma_{s}-1} d x, \text { (19) }
$$

integrate (19) by parts, we get

$\int_{0}^{x} e^{t X^{a}}\left[h_{m}(F(x))-h_{m}(F(y))\right]^{s-r-1} f(x)[F(x)]^{\gamma_{s}-1} d x$

$=\frac{1}{\gamma_{s}}\left[a t \int_{0}^{x} x^{a-1} e^{t X^{a}}[F(x)]^{\gamma_{s}}\right.$

$+\left[h_{m}(F(x))-h_{m}(F(y))\right]^{s-r-1} d x+(s-r-1)$

$\left.\times \int_{0}^{x} e^{t X^{a}}[F(x)]^{\gamma_{s}+m} f(x)\left[h_{m}(F(x))-h_{m}(F(y))\right]^{s-r-2} d x.\right]$.

Substituting (6) and (20) in (19), we get

$$
\begin{aligned}
& M_{X_{(s, n: m: k)}^{(a)} X_{(r, n: m: k)}}(t \mid y)-M_{\left.X_{(s-1, n: m: k)}^{(a)}\right|_{(r, n: m: k)}}(t \mid y) \\
& =\frac{a t C_{s-1}}{\gamma_{s} C_{r-1}(s-r-1) !}[F(y)]^{m-\gamma_{r}+1}
\end{aligned}
$$$$
\times \int_{0}^{x} \frac{x^{a-1}}{\lambda^{\prime}(x)} e^{t X^{a}}[F(x)]^{\gamma_{s}-1}\left[h_{m}(F(x))-h_{m}(F(y))\right]^{s-r-1} f(x) d x,
$$

so, we can rewrite Equation (21) in the form

$$
\begin{aligned}
& M_{X_{(s, n: m: k)}^{(a)} X_{(r, n: m: k)}}(t \mid y)-M_{\left.X_{(s-1, n: m: k)}^{(a)}\right|_{(r, n: m: k)}}(t \mid y) \\
& =\frac{a t}{\gamma_{s}} E\left[\frac{X_{(s, n: m: k)}^{(a-1)} e^{t X_{(s, n: m: k)}^{(a)}}}{\lambda\left(x_{s, n: m: k}\right)} \mid X_{(r, n: m: k)}=y\right],
\end{aligned}
$$

hence, we have the result.

Conversely, if the characterizing condition (18), is satisfied, then from (3) and (17), we have

$\frac{C_{s-1}[F(y)]^{m-\gamma_{r}+1}}{C_{r-1} \Gamma(s-r)}$

$$
\begin{aligned}
& \times \int_{0}^{x} e^{t x^{a}}\left[h_{m}(F(x))-h_{m}(F(y))\right]^{s-r-1} f(x)[F(x)]^{\gamma_{s}-1} d x \\
& =\frac{C_{s-1}[F(y)]^{m-\gamma_{r}+1}}{\gamma_{s} C_{r-1} \Gamma(s-r)}\left[\int_{0}^{x} e^{t x^{a}}[F(x)]^{\gamma_{s}+m}\right. \\
& \times\left[h_{m}(F(x))-h_{m}(F(y))\right]^{s-r-2} f(x) d x \\
& -\int_{0}^{x} \frac{x^{a-1}}{\lambda^{\prime}(x)} e^{t x^{a}}[F(x)]^{\gamma_{s}-1} f(x) . \\
& \left.\times\left[h_{m}(F(x))-h_{m}(F(y))\right]^{s-r-1} d x\right] .
\end{aligned}
$$

Integrating the first integral on the right hand side of Equation (23) by parts, yields

$$
\begin{aligned}
& \frac{C_{s-1}[F(y)]^{m-\gamma_{r}+1}}{C_{r-1} \Gamma(s-r)} \\
& \times \int_{0}^{x} e^{t x^{a}}\left[h_{m}(F(x))-h_{m}(F(y))\right]^{s-r-1}[F(x)]^{\gamma_{s}-1} f(x) d x \\
& =\frac{C_{s-1}[F(y)]^{m-\gamma_{r}+1}}{C_{r-1} \Gamma(s-r)}\left[\int_{0}^{x} a t x^{a-1} e^{t x^{a}}[F(x)]^{\gamma_{s}}\right.
\end{aligned}
$$

$$
\begin{aligned}
& \times\left[h_{m}(F(x))-h_{m}(F(y))\right]^{s-r-1} d x+\int_{0}^{x} e^{t x^{a}}[F(x)]^{\gamma_{s}-1} f(x) \\
& \times\left[h_{m}(F(x))-h_{m}(F(y))\right]^{s-r-1} d x \\
& -\frac{a t}{\gamma_{s}} \int_{0}^{x} \frac{x^{a-1}}{\lambda(x)} e^{t x^{a}}[F(x)]^{\gamma_{s}-1} f(x) \\
& \left.\times\left[h_{m}(F(x))-h_{m}(F(y))\right]^{s-r-1} d x\right]
\end{aligned}
$$

then, after some simplifications, we obtain

$$
\begin{aligned}
& {\left[\frac{a t C_{s-1}[F(y)]^{m-\gamma_{r}+1}}{C_{r-1} \Gamma(s-r)} \int_{0}^{x} x^{a-1} e^{t x^{a}}\left[h_{m}(F(x))-h_{m}(F(y))\right]^{s-r-1}\right.} \\
& \left.\times f(x)[F(x)]^{\gamma_{s}-1}\left[F(x)+\frac{f(x)}{\lambda(x)}\right] d x=0\right]
\end{aligned}
$$


Applying Muntz-Szasz theorm to (25) [8], we get

$$
F(x)=-\frac{1}{\lambda^{\prime}(x)} f(x) .
$$

\subsection{Special cases}

(1) By differentiating both sides of Condition (18) with respect to $t$ and then setting $t=0$, we obtain the following recurrence relation for single moment of dgos:-

$$
\begin{aligned}
& \left.E\left[X_{(s, m: n: k)}^{a} \mid X_{(r, n: m: k)}\right)=y\right] \\
& \left.-E\left[X_{(s-1, m: n: k)}^{a} \mid X_{(r, n: m: k)}\right)=y\right] \\
& =\frac{a}{\gamma_{s}} E\left[\frac{\left.\left.X_{(s-1, m: n: k)}^{(a-1)} \mid X_{(r, n: m: k)}\right)=y\right] .}{\lambda\left(x_{(s, n: m: k)}\right)} .\right.
\end{aligned}
$$

(2) Putting $m=0, k=1$ in (18), we obtain the recurrence relations of reversed order statistics as follows:

$$
\begin{aligned}
& M_{\left.X_{s: n}^{(a)}\right|_{r: n}}(t \mid y)-M_{\left.X_{s-1: n}^{(a)}\right|_{r: n}}(t \mid y) \\
& =\frac{a t}{(n-s+1)} E\left[\frac{X_{s: n}^{(a-1)} e^{t X_{s: n}^{(a)}}}{\lambda\left(x_{s: n}\right)} \mid X_{r: n}=y\right], \\
& E\left[X_{s: n}^{a} \mid X_{r: n}=y\right]-E\left[X_{s-1: n}^{a} \mid X_{r: n}=y\right] \\
& =\frac{a}{(n-s+1)} E\left[\frac{X_{s: n}^{a}}{\left.\lambda X_{s: n}^{\prime}\right)} \mid X_{r: n}=y\right] .
\end{aligned}
$$

(3) Setting $m=-1, k=1$ in (18), we get recurrence relation for single moment of lower record values in the form:

$$
\begin{aligned}
& M_{\left.X_{L(s)}^{(a)}\right|_{L(r)}}(t \mid y)-M_{\left.X_{L(s-1)}^{(a)}\right|_{L(r)}}(t \mid y) \\
& =a t E\left[\frac{X_{L(s)}^{a} e^{t X_{u(s)}^{a}}}{\lambda^{\prime}\left(X_{L(s)}\right)} \mid X_{L(r)}=y\right], \\
& E\left[X_{L(s)}^{a} \mid X_{L(r)}=y\right]-E\left[X_{L(s-1)}^{a} \mid X_{L(r)}=y\right] \\
& =a E\left[\frac{X_{L(s)}^{a}}{\lambda^{\prime}\left(X_{L(s)}\right)} \mid X_{L(r)}=y\right],
\end{aligned}
$$

\section{CHARACTERIZATION BASED ON PRODUCT MOMENT FOR DGOS}

Lemma For $1 \leq r<s \leq n-1, n \geq 2$ and $k=1,2, \ldots$

$E\left(X^{i}(r, n, m, k) X^{j}(s, n, m, k)\right)$

$-E\left(X^{i}(r, n, m, k) X^{j}(s-1, n, m, k)\right)$

$=\frac{-j c_{s-1}}{\gamma_{s} \Gamma(r) \Gamma(s-r)} \int_{0}^{\infty x} \int_{0}^{i} y^{j-1}[F(x)]^{m} f(x) g_{m}^{r-1}[F(x)]$

$\times\left[h_{m}(F(x))-h_{m}(F(y))\right]^{s-r-1}[F(y)]^{\gamma_{s}} d y d x$.

Relation 3 Let $X$ be a random variable, then for integers $a$ such that $a \geq 1$, the following recurrence relation is satisfied iff $X$ has cdf (4).

$E\left(X^{i}(r, n, m, k) X^{j}(s, n, m, k)\right)$

$-E\left[X^{i}(r, n, m, k) X^{j}(s-1, n, m, k)\right]$

$=\frac{j}{\gamma_{s}} E\left[\frac{X^{i}}{\lambda^{\prime}(y)}(r, n, m, k) X^{j-1}(s, n, m, k)\right]$.

Proof: From (30), we get

$E\left[X^{i}(r, n, m, k) X^{j}(s, n, m, k)\right]$

$-E\left(X^{i}(r, n, m, k) X^{j}(s-1, n, m, k)\right)$

$=\frac{-j c_{s-1}}{\gamma_{s} \Gamma(r) \Gamma(s-r)} \int_{0}^{\infty x} \int_{0} x^{i} y^{j-1}[F(x)]^{m} f(x) g_{m}^{r-1}[F(x)]$

$\times\left[h_{m}(F(x))-h_{m}(F(y))\right]^{s-r-1}[F(y)]^{\gamma_{s}-1}\left[\frac{-1}{\lambda^{\prime}(y)}\right] f(y) d y d x$,

and hence (31) obtained.

Conversely, if the characterizing condition (4.2) holds, then from (3) and (6), we have

$$
\begin{aligned}
& \frac{C_{s-1}}{\Gamma(r) \Gamma(s-r)} \int_{0}^{\infty x} \int_{0}^{i} x^{j} y^{j}\left[(F(x)]^{m} f(x) g_{m}^{r-1}[F(x)]\right. \\
& \left.\times h_{m}(F(y))-h_{m}(F(x))\right]^{s-r-1}(F(y))^{\gamma_{r}-1} f(y) d y d x \\
& =\frac{C_{s-2}}{\Gamma(r) \Gamma(s-r)} \int_{0}^{\infty} \int_{0}^{x} x^{i} y^{j}\left[(F(x)]^{m} f(x) g_{m}^{r-1}[F(x)]\right. \\
& \left.\times h_{m}(F(y))-h_{m}(F(x))\right]^{s-r-2}(F(y))^{\gamma_{r}+m} f(y) d y d x
\end{aligned}
$$


$+\frac{j C_{s-1}}{\gamma_{s} \Gamma(r) \Gamma(s-r)} \int_{0}^{\infty} \int_{0}^{x} \frac{x^{i}}{\lambda^{i}(y)} y^{j-1}\left[(F(x)]^{m} f(x)\right.$

$\times g_{m}^{r-1}[F(x)]\left[h_{m}(F(y))-h_{m}(F(x))\right]^{s-r-1}$

$\times(F(y))^{\gamma_{r}-1} f(y) d y d x$.

Integrating the first integral on the right hand side of Equation (33) by parts, we get

$$
\begin{aligned}
& \int_{0}^{\infty} y^{j}\left[h_{m}(F(y))-h_{m}(F(x))\right]^{s-r-2}(F(y))^{\gamma_{r}+m} f(y) d y \\
& =\frac{j}{(s-r-1)} \int_{0}^{\infty} y^{j-1}\left[h_{m}(F(y))-h_{m}(F(x))\right]^{s-r-1} \\
& \times(F(y))^{\gamma_{r}} d y+\frac{\gamma_{s}}{(s-r-1)} \\
& \times \int_{0}^{\infty} y^{j}\left[h_{m}(F(y))-h_{m}(F(x))\right]^{s-r-1}(F(y))^{\gamma_{r}-1} f(y) d y .
\end{aligned}
$$

Substituting (34) in (33), we get

$$
\begin{aligned}
& \frac{j C_{s-1}}{\gamma_{s} \Gamma(r) \Gamma(s-r)} \int_{0}^{\infty x} \int_{0} x^{i} y^{j-1}\left[(F(x)]^{m} f(x) g_{m}^{r-1}[F(x)]\right. \\
& \left.\times h_{m}(F(y))-h_{m}(F(x))\right]^{s-r-1}[F(y)]^{\gamma_{s}-1} \\
& \times\left\{F(y)+\frac{1}{\lambda^{\prime}(y)} f(y)\right\} d y d x=0
\end{aligned}
$$

Now applying a generalization of the Muntz-Szasz Theorm [8], we get

$$
F(y)=-\frac{1}{\lambda^{\prime}(y)} f(y), y \geq 0 .
$$

\subsection{Special Cases}

(1) Putting $m=0, k=1$ in (31), we obtain the recurrence relations of reversed order statistics as follows:

$$
\mu_{r: n}^{(a)}-\mu_{r-1: n}^{(a)}=\frac{j}{n-s+1} E\left[\frac{X_{r: n}^{i}}{\lambda(y)} X_{s: n}^{j-1}\right] .
$$

(3) Setting $m=-1, k=1$ in (31), we get recurrence relation for single moment of lower record values in the form:

$$
\begin{aligned}
& E\left(X^{i}(r, n, m, k) X^{j}(s, n,-1,1)\right) \\
& -E\left[X^{i}(r, n, m, k) X^{j}(s-1, n,-1,1)\right]
\end{aligned}
$$

$=j E\left[\frac{X^{i}}{\lambda^{\prime}(y)}(r, n,-1,1) X^{j-1}(s, n,-1,1)\right]$.

\section{THE INVERSE WEIBULL DISTRIBUTION}

The distribution function of the inverse Weibull distribłfighn is given by:

$$
F(x \mid \theta)=\exp \left[-(\alpha x)^{-\beta}\right], \quad x>0,
$$

where $\theta=(\alpha, \beta), \alpha>0, \beta>0$ and

$$
\lambda(x)=\frac{\alpha^{-\beta}}{x^{\beta}}, \lambda^{\prime}(x)=-\frac{\beta \alpha^{-\beta}}{x^{\beta+1}} .
$$

From Equations (14), (27) and (31), we have the following relations:

$$
\begin{aligned}
& \mu_{r ; n: m: k}^{(a)}-\mu_{r-1 ; n: m: k}^{(a)}=\frac{-a \alpha^{\beta}}{\beta \gamma_{r}} E\left[X_{r, n: m: k}^{(a+\beta)}\right] . \\
& \left.E\left[X_{(s, m: n: k)}^{a} \mid X_{(r, n: m: k)}\right)=y\right] \\
& \left.-E\left[X_{(s-1, m: n: k)}^{a} \mid X_{(r, n: m: k)}\right)=y\right] \\
& \left.=\frac{-a \alpha^{\beta}}{\beta \gamma_{s}} E\left[X_{(s, m: n: k)}^{(a+\beta)} \mid X_{(r, n: m: k)}\right)=y\right] . \\
& E\left(X^{i}(r, n, m, k) X^{j}(s, n, m, k)\right) \\
& -E\left[X^{i}(r, n, m, k) X^{j}(s-1, n, m, k)\right] \\
& =\frac{-j \alpha^{\beta}}{\beta \gamma_{s}} E\left[X^{i}(r, n, m, k) X^{j+\beta}(s, n, m, k)\right] .
\end{aligned}
$$

\section{CONCLUSION}

The recurrence relations for single and product moments based on moment generating function of dual generalized order statistics from the inverse exponential-type distribution are obtained. The recurrence relation for the single moments is used to characterize the inverse exponential-type distribution. Recurrence relations for single and product moments of reversed order statistics and Power record values are deduced as special cases. All results can be applied to many inverse distribution such as inverse exponential, inverse Rayleigh and inverse Weibull.

\section{ACKNOWLEDGMENTS}

The authors would like to express their thanks to the referees and the Editor for their helpful remarks that led to improve the original manuscript. 


\section{REFERENCES}

[1] Kumer, D. (2011). Generalized order statistics from kumaraswamy distributibution and its characterization, Journal of Information and Mathematical Sciences, 27, 4, 463-476.

[2] Khan, U. and Kumer, D. (2010). On moments of lower generalized order statistics from exponentiated Pareto distribution and its characterization, Journal of applied Mathematical Sciences, 4, 2711-2722.

[3] Mbah, A. K. and Ahsanullah, M. (2007). Some characteriztion of the power function distribution based on lower generalized order statistics, Pakistan Journal of Statistics, 23, 139-146.

[4] Al-Hussaini, E. K., Ahmad, A. A. and ElBoughdady, H. H. (2004). Recurrence relations for moment generating functions of order statistics, Metron, IXII, 85-99.
[5] Pawlas, P. and Szynal, D. (2001). Recurrence relations for single and product moments of lower generalized order from the inverse Weibull distribution, Journal of Demonstratio Mathematica, 34-2, 2, 353-358.

[6] Khan, R. U., Anwar, Z. and Athar, H. (2008) Recurrence relations for single and product moments of dual generalized order statistics from exponentiated Weibull distribution, Aligarh journal of Statistics, 28,37-45.

[7] Al-Hussaini, E. K., Ahmad, A. A. and Al-Kashif, M. A. (2005). Recurrence relations for moment and conditional moment generating functions of generalized order statistics, Metrika, 61, 2, 199-220.

[8] Hwang, J. S. and Lin, G. D. (1984). On a generalized moments problem II, Journal ofProceedings American Mathematical Society, 91 577-580. 